\title{
Intramolecular Electron Scattering and Electron Transfer Following Autoionization in Dissociating Molecules
}

\author{
O. Kugeler, ${ }^{1}$ G. Prümper, ${ }^{1, *}$ R. Hentges,${ }^{1}$ J. Viefhaus, ${ }^{1}$ D. Rolles, ${ }^{1}$ U. Becker, ${ }^{1}$ S. Marburger, ${ }^{2}$ and U. Hergenhahn ${ }^{2, \dagger}$ \\ ${ }^{1}$ Fritz-Haber-Institut der Max-Planck-Gesellschaft, Faradayweg 4-6, 14195 Berlin, Germany \\ ${ }^{2}$ Max-Planck-Institut für Plasmaphysik, Boltzmannstrasse 2, 85748 Garching, Germany
}

(Received 6 April 2004; published 16 July 2004)

\begin{abstract}
Resonant Auger decay of core-excited molecules during ultrafast dissociation leads to a Doppler shift of the emitted electrons depending on the direction of the electron emission relative to the dissociation axis. We have investigated this process by angle-resolved electron-fragment ion coincidence spectroscopy. Electron energy spectra for selected emission angles for the electron relative to the molecular axis reveal the occurrence of intermolecular electron scattering and electron transfer following the primary emission. These processes amount to approximately $25 \%$ of the resonant atomic Auger intensity emitted in the studied transition.
\end{abstract}

DOI: 10.1103/PhysRevLett.93.033002

The fast dissociation of excited diatomic molecules, such as $\mathrm{O}_{2}$, allows for an interesting case study of symmetry breaking in a homonuclear molecule. By angleresolved coincident detection of autoionization electrons and fragment ions, we were able to examine details of the intramolecular relaxation dynamics that are hidden to noncoincident spectroscopy. This study was motivated by the wish to observe processes relevant for chemical reactions on their natural time scale, on which very little information is available.

Among the fastest processes with respect to the nuclear dynamics involved is the fragmentation of a small molecule, which is initiated when a core electron is resonantly excited into an empty antibonding valence state. By that, dissociation of the nuclear framework and relaxation of the electronic shell are induced; they compete on a similar, femtosecond time scale. After discovery of this time scale, termed "core-hole clock" [1], interference effects of the outgoing nuclear wave packets $[2,3]$ and most recently the momentum exchange of the fast accelerating fragments with the atomic autoionization electrons (also called resonant Auger electrons) [4,5] have been investigated.

When the nuclear dynamics proceeds fast enough, a fraction of the molecules is already dissociated when autoionization takes place. This is the case for the deexcitation of the $\mathrm{O}_{2} 1 s \rightarrow 3 \sigma_{u}^{*}$ resonance [6], investigated in this work. A schematic representation of the potential curves relevant in this decay process is shown in Fig. 1. In the following we concentrate on the autoionization electrons that are emitted in the atomic region of the potential curve. Since we have excited a homonuclear diatomic molecule, both core-hole and excited electrons are the result of excitation from delocalized molecular orbitals of $g$ or $u$ symmetry. In this case, they still belong to both of the fragments, resembling an entangled state, with respect to the correlation of the momentum of the atomic cores and the electron momentum vector. An
PACS numbers: 33.80.Eh, 33.15.Vb, 34.20.Gj, 34.80.Bm

atomic autoionization electron is eventually emitted from only one of the atoms. These electrons experience a shift of their momenta, depending on their emission direction relative to the velocity vector of the emitting fragment. An energy splitting in the autoionization electron spectrum associated with the different emission directions has been demonstrated by Björneholm et al. [4] and was termed Doppler shift. Björneholm et al., however, recorded only the energy of the resonant Auger electrons emitted on the $\mathrm{O}_{2} 1 s \rightarrow 3 \sigma_{u}^{*}$ resonance $[4,7]$. To relate the energy shifts in the resonant Auger spectrum to the emission direction of the electrons with respect to the molecular axes was possible only since, because of the dipole selection rules on a $\sigma$-type resonance, the molecules are preferentially excited with their axis orientation along the electric dipole vector of the radiation. A more detailed insight into the decay process

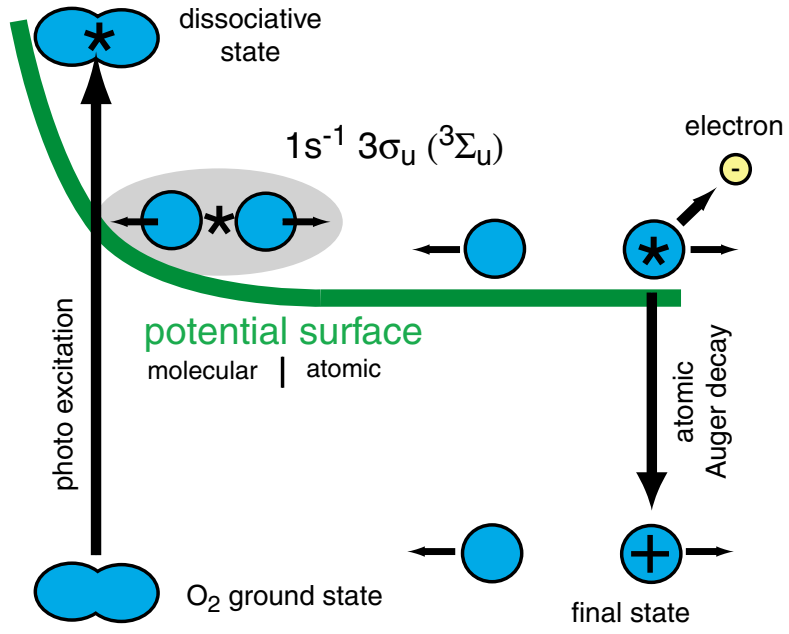

FIG. 1 (color). Schematic of the $\mathrm{O}_{2} 1 s \rightarrow 3 \sigma_{u}^{*}$ resonant excitation and its decay. Because of the highly repulsive intermediate potential surface, excited $\mathrm{O}^{*}$ fragments may reach the atomic region before they deexcite. 
is possible in an experiment where the axis orientation of each molecule is probed individually. We have set up an apparatus, in which electrons are detected with high resolution in a hemispherical energy analyzer, while ions are projected into a time-of-flight spectrometer with a position sensitive anode, and are recorded in coincidence with the electrons. In this Letter, we show that a significant fraction of the autoionization electrons emitted in the atomic region undergoes scattering on the other, neutral fragment atom after emission and that electron transfer between the two fragments is possible even after dissociation and autoionization, which is already in the atomic region.

The electron detection part of the experimental setup (see upper half of Fig. 2) consists of a hemispherical electron spectrometer (Scienta ES200), modified by us with a fast, position resolving electron detector [8]. The analyzer is mounted in the plane perpendicular to the light propagation axis and under $54.7^{\circ}$ with respect to the storage ring plane. A projecting ion spectrometer [9] is placed opposite to the electron spectrometer. The use of pulsed fields in the ion spectrometer enables coincidence experiments with high electron energy resolution. For the experiments reported here, the analyzer was operated at a pass energy of $40 \mathrm{eV}$, resulting in an energy resolution better than $100 \mathrm{meV}$. The ion extraction pulse was triggered by the arrival of the electron in the Scienta analyzer, and had an amplitude of $+460 \mathrm{~V}$ at the pusher plate and $-680 \mathrm{~V}$ at the drift tube, a rise time of $15 \mathrm{~ns}$, and an insertion delay of $468 \mathrm{~ns}$. The extractor plate was grounded. All events were stored separately for off-line data analysis. Reconstruction of the ion momenta was done according to a look-up table produced by a numerical simulation of the ion trajectories. Fragments with up to $10 \mathrm{eV}$ kinetic energy were detected with a $4 \pi$ solid angle. We have determined the maximum of the fragment

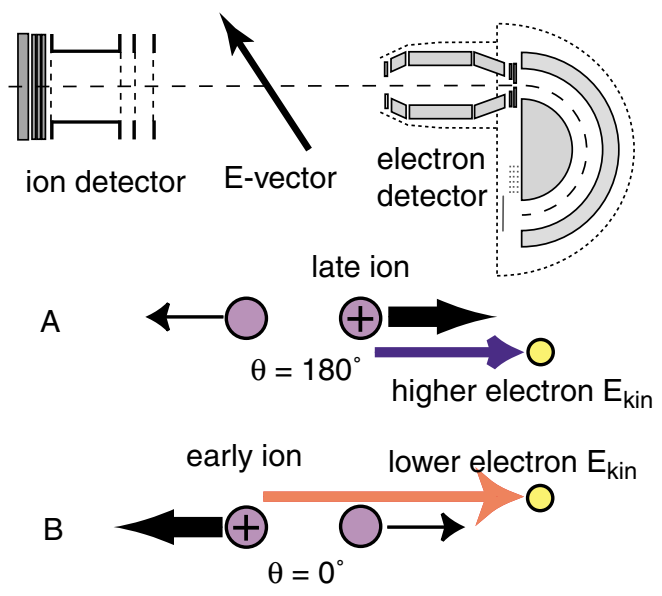

FIG. 2 (color). Experimental layout and kinematical correlation between electron and ion momenta. All ions, but only electrons emitted in the direction of the hemispherical analyzer, are detected. energy distribution as $3.5 \pm 0.5 \mathrm{eV}$. Photons were provided by the U49/2-PGM1 beam line of the BESSY synchrotron radiation source (Berlin, Germany). A suggestion of this principal setup has been made earlier [10].

The lower part of Fig. 2 schematically displays the kinematics of the process. Electrons displaced to smaller energies (redshift) are expected to be emitted counterparallel to the fragment ion velocity. Since the ion detector opposes the electron spectrometer, ions from these events have the shortest time of flight to the detector, corresponding to $\theta=0^{\circ}$, and are termed "early" ions in Fig. 2. Ions with a longer time of flight stem from molecules, which have fragmented into a direction with a larger $\theta$, denoted " $B$ " in Fig. 2. Parallel emission of electron and ion corresponds to $\theta=180^{\circ}$, denoted " $A$." Thus, the ion time of flight is at the same time a measure for the relative angle between the directions of fragment and electron momentum. Figure 3 shows the relation between electron energy and ion time of flight for a total of 52000 coincidence events. The effect of 13000 random coincidences was subtracted using random triggerion events. The collected electron spectrum, summed up over all ion flight times in the top panel of Fig. 3, does not show the double maximum structure observed earlier $[4,7]$, since our electron analyzer is not aligned along the horizontal but along the magic angle. The color coded two-dimensional coincidence histogram in Fig. 3 nevertheless allows us to unequivocally correlate "early ions" to the "redshifted" electrons. The opposite is true for the

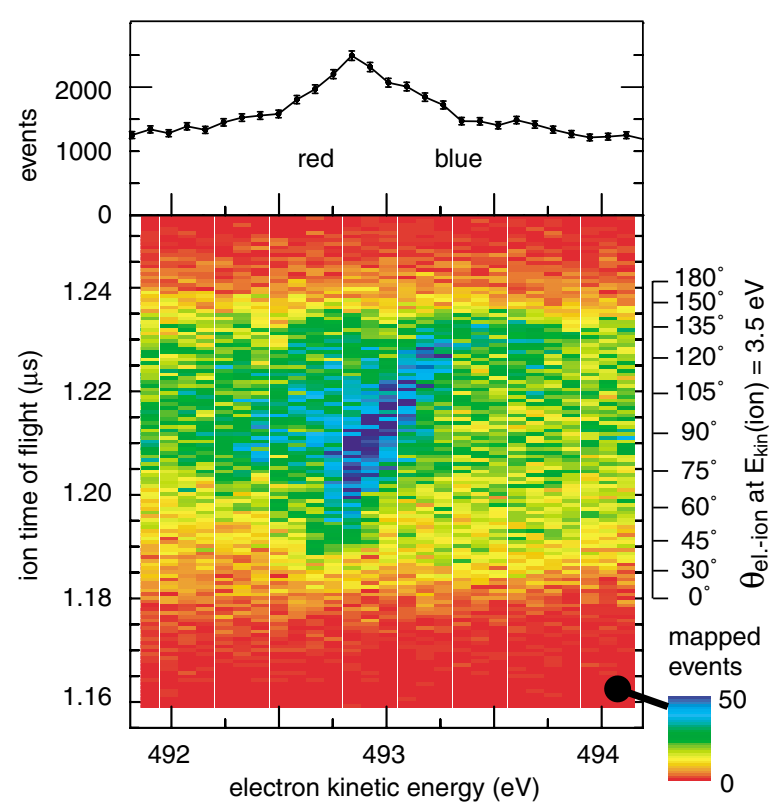

FIG. 3 (color). Main panel: Histogram of autoionization electron, fragment $\mathrm{O}^{+}$ion pairs detected in coincidence. Ion emission angles with respect to the spectrometer axis $\theta$ are given for fragments with a kinetic energy of $3.5 \mathrm{eV}$. Top panel: Electron kinetic energy distribution summed over ion time of flight. 
"late" ions emitted towards the electron spectrometer, which are correlated with electrons shifted to higher energies, the so-called "blueshifted" electrons. The asymmetry in the angle scale is due to the nonlinearity in the relation between the axial component of the ion momentum and the time of flight. This also implies a small deviation from the intuitively expected mirror symmetry to the $90^{\circ}$ axis.

For a more thorough analysis, we have sorted the events into ten (partially overlapping) subsets according to ten different angle intervals between the fragmentation direction and the electron emission direction. The electron kinetic energy distribution curves of the two subsets of events, in which the electron was emitted into a cone of $45^{\circ}$ opening angle and aligned parallel or antiparallel to the direction of the fragment that was eventually detected, respectively, are displayed in Fig. 4. The other subsets were $30^{\circ}-55^{\circ}, 45^{\circ}-70^{\circ}, 70^{\circ}-80^{\circ}$, and $80^{\circ}-90^{\circ}$, and their respective counterparts. They have been used in the analysis delineated below.

According to the simple picture for the process given by Björneholm et al. in each panel of Fig. 4, only one peak should appear. For electron emission roughly in the direction of the ion velocity $\left(\theta>90^{\circ}\right)$ this peak should be shifted towards higher kinetic energy, and vice versa for emission of the electron opposite to the ion direction $(\theta<$ $90^{\circ}$ ). These features, labeled $A$ and $B$ in Fig. 4, can indeed be identified, but additional structures are also present in both panels. Prominent is a peak $\left(C_{\mathrm{B}}\right)$ occurring for parallel (ion, electron) emission, but at a shift towards low kinetic energies that far exceeds the shift found for antiparallel emission. We attribute this peak to the backscattering of an electron at the neutral fragment atom. For electrons being initially emitted exactly along the molecular axis we have calculated the velocity after this elastic scattering by classical mechanics as

$$
v^{\prime}(\theta)=\sqrt{v^{2}-4 u v+5 u^{2}+2 u(v-2 u) \cos \theta},
$$

where $u$ is the fragment velocity of one oxygen atom in the laboratory frame, and $v$ is the initial velocity of the electron without Doppler shift. For backscattering $(\theta=$ $180^{\circ}$ ) this gives an energy shift of 3 times the Doppler shift of the unscattered electrons, which is very close to the value we observe. The remaining energy difference can easily be explained by the fact that we record electrons with some distribution of relative emission angles. Forward scattered electrons $\left(C_{\mathrm{F}}\right)$ are expected to energetically coincide with redshifted electrons $(B)$ at the shown angular selection.

Additional to the scattered intensities, both panels show the presence of electrons with a Doppler shift that would fit to a direction of the ion momentum just opposite to what is observed $\left(A^{*}\right.$ and $\left.B^{*}\right)$. That is, the upper panel, where the ion has its original momentum in the direction of the electron detector, shows the presence of electrons

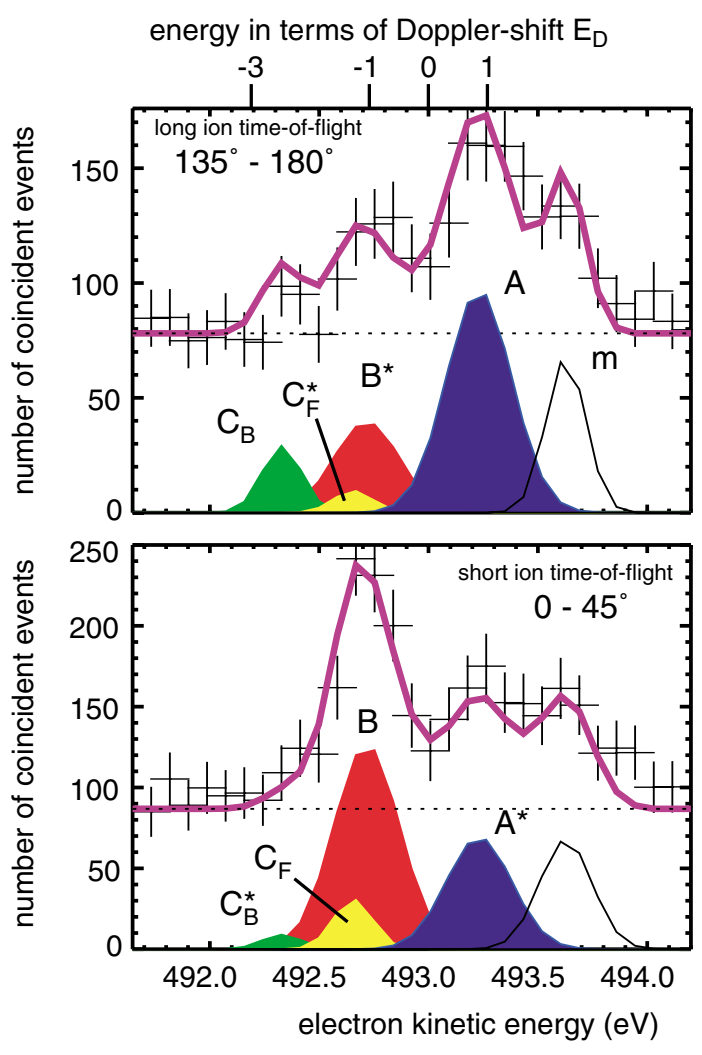

FIG. 4 (color). Kinetic energy spectra of electrons emitted parallel and antiparallel to the ions at relative angles of $135^{\circ}-$ $180^{\circ}$ and $0-45^{\circ}$, respectively. The solid curves represent a fit result based on all available relative angles. Components $A$ and $B$ correspond to resonant Auger emission in the atomic region, components $A^{*}$ and $B^{*}$ to processes, in which after the electron emission a charge transfer to the ionic fragment occurs, components $C_{\mathrm{B}}$ and $C_{\mathrm{F}}$ to backward and forward scattering of resonant Auger electrons on the neutral fragment, and $C_{\mathrm{B}}^{*}$ and $C_{\mathrm{F}}^{*}$ to processes involving energy exchange by scattering and charge exchange between the fragments. The peak labeled " $m$ " we assign to decay into a molecular ionic state occurring before dissociation.

with a Doppler shift characteristic for emission counterparallel to the ion velocity, that is, in the direction of the ion spectrometer. Since our electron spectrometer accepts only electrons emitted within a small cone, these events cannot belong to electrons emitted by the ion that is detected. We are therefore led to conclude that this additional intensity results from an exchange of a negative charge between the two oxygen fragments after the atomic resonant Auger electron has been emitted.

To gather further evidence for this explanation of the spectral features in the angle-selected event subsets, we have attempted their quantitative reproduction by a fit guided by the following assumptions.

(1) Every spectrum contains four peaks, pertaining to direct emission into the detector ( $A$ or $B$, respectively), emission after scattering on the neutral fragment $\left(C_{\mathrm{B}}\right.$ or $C_{\mathrm{F}}$ ), emission into the detector with subsequent charge 
transfer between the fragments $\left(B^{*}\right.$ or $\left.A^{*}\right)$, and a scattered peak with subsequent charge transfer $\left(C_{\mathrm{B}}^{*}\right.$ or $\left.C_{\mathrm{F}}^{*}\right)$.

(2) The area of the charge transfer peak is a constant fraction of the area of the respective direct peaks, for all five subsets of events, $A^{*}=c A, B^{*}=c^{\prime} B$. The constants have been chosen differently in order to account for a possible anisotropy in the charge transfer after parallel and antiparallel emission of the autoionization electron. The difference between $c$ and $c^{\prime}$ turns out to be less than $10 \%$.

(3) The sum of redshifted and scattered electrons equals the number of blueshifted electrons, $A=$ $B+C_{\mathrm{B}}+C_{\mathrm{F}}$.

(4) The positions of all peaks in each angle selection are fixed to values derived by Eq. (1).

(5) The peaks are modeled by Gaussians of constant width, and a constant background is added.

(6) The intensity from molecular resonant Auger decay indicated as " $m$," which appears in all of the spectra, was modeled by an additional line of fixed kinetic energy.

Using a random walk method, we thus arrive at the decomposition shown by the colored areas in Fig. 4. Decompositions of a comparable quality for other angle selected subsets are not displayed here.

Very recently, intramolecular electron scattering in dissociating molecules has been theoretically considered [11]. The appearance of an additional peak due to energy loss by backscattering at the fast neutral fragment was predicted. We found this additional peak at the predicted position. Somewhat different from our approach, this peak is seen as a special case of double scattering, and is predicted to appear only in a very small range of $\theta=$ $180^{\circ}$ angles. For $\theta=180^{\circ}$ the backscattered electron passes the emitting ions in a close distance, so that the notion of double scattering is, in fact, justified. However, for $\theta$ values close to $180^{\circ}$ the momentum transfer of the second scattering process can be neglected reducing the kinematical discussion to Eq. (1).

A consequence of the intramolecular scattering we describe here is that the fraction of electrons detected traveling in the direction of the ions is always larger than the fraction of electrons detected in the opposite direction. This intrinsic asymmetry with respect to emission into the inner and outer regions of the molecule explains asymmetric electron emission behavior in diatomic homonuclear molecules [12], which was for a long time not understood well, because it was in distinct contrast to results on the same subject obtained by soft $\mathrm{x}$-ray experiments instead of electron emission [13]. Such energy resolved coincidence spectroscopy of intramolecular scattering processes has a great potential for time-dependent studies of dissociating molecular systems. This is particularly true when pump-probe experiments with free electron lasers become feasible. But already today, subtle insight into the dynamics of poly- atomic dissociating molecules, such as $\mathrm{SF}_{6}$, are possible [14]. An analogous experiment on $\mathrm{SF}_{6}$ performed at HASYLAB (DESY) shows results equivalent to $\mathrm{O}_{2}$ with respect to the electron energy shifts, as exhibited in Fig. 3. Turning to coincidences with particular ionic fragments reveals, however, an even richer picture. Besides electron backscattering, the signature of another process, closely related to the symmetric charge transfer in $\mathrm{O}_{2}$ is found: After autoionization of the fluor atomic fragment, an asymmetric electron transfer from the other $\mathrm{SF}_{n}$ fragment may neutralize this cation. The measured spectral changes and even more their angular distributions provide a means for the qualitative and eventually quantitative analysis of these processes and the conformational changes occurring during the excitation [15] and the breakup of the molecule. In the long term, the method may become a major tool for time-resolved photoelectron diffraction studies.

We acknowledge the contribution of F. Burmeister in data acquisition. This project was partially funded by the Deutsche Forschungsgemeinschaft under Grants No. He 3060/4-1 and No. Be 860/18-2.

*Present address: Institute of Multidisciplinary Research for Advanced Materials, Tohoku University, Sendai 9808577, Japan.

${ }^{\dagger}$ Also at BESSY GmbH, Albert-Einstein-Straße 15, 12489 Berlin, Germany.

Electronic address: uwe.hergenhahn@ipp.mpg.de

[1] O. Björneholm et al., Phys. Rev. Lett. 79, 3150 (1997).

[2] E. Pahl, L. S. Cederbaum, H.-D. Meyer, and F. Tarantelli, Phys. Rev. Lett. 80, 1865 (1998).

[3] R. Feifel et al., Phys. Rev. Lett. 85, 3133 (2000).

[4] O. Björneholm et al., Phys. Rev. Lett. 84, 2826 (2000).

[5] K. Ueda et al., Phys. Rev. Lett. 90, 233006 (2003).

[6] C. D. Caldwell, S. J. Schaphorst, M. O. Krause, and J. Jiménez-Mier, J. Electron Spectrosc. Relat. Phenom. 67, 243 (1994).

[7] A. Baev, F. Gel'mukhanov, P. Sałek, H. Ågren, K. Ueda, A. de Fanis, K. Okada, and S. Sorensen, Phys. Rev. A 66, 022509 (2002).

[8] O. Kugeler, S. Marburger, and U. Hergenhahn, Rev. Sci. Instrum. 74, 3955 (2003).

[9] U. Becker, O. Gessner, and A. Rüdel, J. Electron Spectrosc. Relat. Phenom. 108, 189 (2000).

[10] F. Gel'mukhanov and H. Ågren, JETP Lett. 67, 1064 (1998).

[11] F. Gel'mukhanov, V. Kimberg, and H. Ågren, Phys. Rev. A 69, 020501(R) (2004).

[12] A.V. Golovin et al., Phys. Rev. Lett. 79, 4554 (1997); A. Lafosse et al., J. Chem. Phys.. 114, 6605 (2001).

[13] P. Glans et al., Phys. Rev. Lett. 76, 2448 (1996).

[14] M. Kitajima et al., Phys. Rev. Lett. 91, 213003 (2003).

[15] L. Lehr, M.T. Zanni, C. Frischkorn, R. Weinkauf, and D. M. Neumark, Science 284, 635 (1999) 